Draft VERSION JANUARY 16, 2020

Typeset using $\mathrm{LAT}_{\mathrm{E}} \mathrm{X}$ twocolumn style in AASTeX63

\title{
Dynamical structure of small bulges reveals their early formation in $\Lambda$ CDM paradigm
}

\author{
Luca Costantin, ${ }^{1,2}$ Jairo Méndez-Abreu, ${ }^{3,4}$ Enrico M. Corsini,${ }^{5,}{ }^{6}$ Lorenzo Morelli,${ }^{7}$ \\ Adriana de Lorenzo-Cáceres,${ }^{3,4}$ Ilaria Pagotto, ${ }^{8}$ Virginia Cuomo, ${ }^{5}$ J. Alfonso L. Aguerri, ${ }^{3,4}$ And \\ Michela Rubino 5 \\ ${ }^{1}$ Centro de Astrobiología (CSIC-INTA), Ctra de Ajalvir km 4, Torrejón de Ardoz, E-28850 Madrid, Spain \\ ${ }^{2}$ INAF - Astronomical Observatory of Brera, via Brera 28, I-20121 Milano, Italy \\ ${ }^{3}$ Instituto de Astrofísica de Canarias, Calle Va Láctea s/n, E-38200 La Laguna, Tenerife, Spain \\ ${ }^{4}$ Departamento de Astrofísica, Universidad de La Laguna, Calle Astrofísico Francisco Sánchez s/n, E-38205 La Laguna, Tenerife, Spain \\ ${ }^{5}$ Dipartimento di Fisica e Astronomia "G. Galilei", Università di Padova, vicolo dell'Osservatorio 3, I-35122, Padova, Italy \\ ${ }^{6}$ INAF - Osservatorio Astronomico di Padova, vicolo dell'Osservatorio 5, I-35122 Padova, Italy \\ ${ }^{7}$ Instituto de Astronomía y Ciencias Planetarias, Universidad de Atacama, Copayapu 485, Copiapó, Chile \\ ${ }^{8}$ Leibniz-Institut für Astrophysik Potsdam (AIP), An der Sternwarte 16, D-14482 Potsdam, Germany
}

\begin{abstract}
The $\Lambda$ cold dark matter $(\Lambda \mathrm{CDM})$ paradigm of galaxy formation predicts that dense spheroidal stellar structures invariably grow at early cosmic time. These primordial spheroids evolve toward a virialized dynamical status as they finally become today's elliptical galaxies and large bulges at the center of disk galaxies. However, observations reveal that small bulges in spiral galaxies are common in the nearby universe. The prevailing belief that all small bulges form at later times from internal processes occurring in the disk represents a challenge for the $\Lambda \mathrm{CDM}$ scenario. Notably, the coevolution of bulges and central supermassive black holes (SMBHs) at early phases of galaxy evolution is also at stake. However, observations have so far not provided conclusive evidence against their possible early origin. Here, we report new observations of small bulges showing that they follow the massvelocity dispersion relation expected for virialized systems. Contrary to previous claims, small bulges bridge the gap between massive ellipticals and globular clusters. This dynamical picture supports a scenario where systems over seven orders of magnitude in stellar mass form at early cosmic time. These results alleviate the tension between $\Lambda \mathrm{CDM}$ simulations and observations at galactic scales. We hypothesize that these small bulges are actually the low-mass descendants of compact objects observed at high redshift, also known as red nuggets, which are consistently produced in cosmological $\Lambda \mathrm{CDM}$ simulations. Therefore, this also suggests that the established coevolution of SMBHs and large bulges naturally extends to spheroids in the low-mass regime.
\end{abstract}

Keywords: galaxies: bulges — galaxies: evolution — galaxies: formation — galaxies: fundamental parameters - galaxies: kinematics and dynamics

\section{INTRODUCTION}

The $\Lambda \mathrm{CDM}$ model is very successful at reproducing observations of the large-scale structures of the universe in a cosmological context. In this scenario, dark matter halos initially convert their potential energy into kinetic energy reaching a virialized status, allowing then galaxies to form due to the cooling of baryons (White \& Rees 1978). However, the evolution of luminous structures

Corresponding author: Luca Costantin

l.costantin@cab.inta-csic.es is observed to markedly deviate from the assembly history of dark matter halos, challenging our understanding of the interplay between baryonic structures and the dark matter content in the universe (Bullock \& BoylanKolchin 2017).

The degree by which galaxies satisfy fundamental scaling relations likely reflects the underlying dynamical principles of how baryons settle within dark matter potential wells. The theoretical prediction from the Virial Theorem is that the photometric and kinematic information of virialized systems are coupled in a way that they tend to occupy a preferential position in fundamental 
scaling relations (e.g., Fundamental Plane; Djorgovski \& Davis 1987).

In this context, the brightest and most massive earlytype galaxies (ETGs) were the first systems observed to satisfy the fundamental scaling relations valid for virialized systems. The evolution of ETGs relies on the socalled two-phase formation scenario (Oser et al. 2010). In the first stage, the galaxy forms the bulk of its stellar component experiencing a gas-rich dissipational collapse within dark matter halos. In the second stage, from redshift $z \lesssim 2$, dry accretion of satellites and occasional major mergers drive their evolution. Regardless of the mechanisms that funnel the gas to the center of the dark halos (e.g., disk instabilities, accretion from cosmic web, or wet mergers), the initial phase of galaxy formation leads to a dense stellar core in a rapid formation process, known as gas compaction (Dekel et al. 2009). The build-up of the core is regulated by the growth of the central supermassive black hole (SMBH) that coevolves with it (Silk \& Rees 1998). This process, which takes place at high redshift, sets the overall properties of massive ETGs, which ultimately translate into the scaling relations observed in the local universe after the system virialization process.

The compelling evidence that large bulges swelling out the center of massive spiral galaxies satisfy the same fundamental scaling relations of ETGs leads us to infer that they share a similar formation process. This scenario implies an early formation of the bulge, prior to the disk, that would be instead strongly perturbed if not destroyed in case of a later formation. Moreover, observations of SMBHs ubiquitously residing in all massive bulges, with bigger black holes living in larger bulges (Ferrarese \& Merritt 2000), lead to the belief that black hole growth and bulge formation undeniably regulate each other. Interestingly, some authors have proposed that SMBHs do not coevolve with dark matter, disks, or disk-like structures within galaxies (Kormendy et al. 2011; Kormendy \& Bender 2011).

In late-type galaxies secular processes connected to disk evolution are thought to be responsible for building their small and less massive bulges (Kormendy \& Kennicutt 2004). These continuous and slow processes are thought to shape the overall physical properties of the central region of late-type galaxies into a disk-like structure (Kormendy \& Fisher 2008, but see Guo et al. 2020 for the build-up of non-disky bulges out of internal secular evolution). While ETGs virialized out of the density fluctuations of cold dark matter (CDM) in short timescales, slow processes responsible for building up disk-like systems prevent them from reaching a virialized status. Thus, the severe implication is that the proposed disky nature of small bulges implies that they have to deviate from the fundamental virial relations valid for ETGs and massive bulges (Fisher \& Drory 2016). Moreover, the internal redistribution of angular momentum in the disk-like component and the gradual gas infall provide less black hole $(\mathrm{BH})$ feeding and may drive slower SMBH growth. Therefore, the supposed late and slow formation of small bulges in latetype galaxies fuels the tension between $\mathrm{SMBH}$ and bulge coevolution (Kormendy et al. 2011; Kormendy \& Bender 2011).

\section{SAMPLE AND OBSERVATIONS}

New spectroscopic observations of a sample of 26 extremely late-type spiral galaxies allow us to describe the dynamical status of small bulges in the context of $\Lambda \mathrm{CDM}$ paradigm.

The sample galaxies have been selected from the Sloan Digital Sky Survey (SDSS) Data Release 13 spectroscopic catalog (Albareti et al. 2017), which provides a unique framework for statistical galaxy studies. They are a representative subsample $(\sim 10 \%)$ of a volume limited sample of 247 galaxies down to $M_{r}<-18$ mag located in a volume of $100 \mathrm{Mpc}$ radius. They are defined as late-type according to: $(i)$ concentration $C=R_{90} / R_{50}<2.5$, typical of late-type disk galaxies; (ii) Petrosian radius $R_{\text {petro }}>10^{\prime \prime}$, avoiding extremely small galaxies where spatial resolution problems could arise. They also present: (iii) inclination $i<65^{\circ}$ and $(i v)$ central average surface brightness in $r$-band $\mu_{r}\left(r<2^{\prime \prime}\right)<20$ mag $\operatorname{arcsec}^{-2}$, allowing for a good photometric definition of the half-light radius of the bulge and limiting the effects of dust lanes.

The spectroscopic observations were carried out in two separate observing runs on 2018 February 1316 (A36TAC_11/2017B; PI: L. Costantin) and 2018 May 21-24 (CAT18A_61/2018A; PI: J. Méndez-Abreu). We used the DOLORES spectrograph at the Telescopio Nazionale Galileo in La Palma (Spain) mounting the V510 grism, covering the wavelength range $4875-5325 \AA$ using the $1^{\prime \prime}$ wide slit. The spectrograph was equipped with a E2V 4240 camera and a thinned back-illuminated, deep-depleted, Astro-BB coated charge-coupled device $(\mathrm{CCD})$ with $2048 \times 2048$ pixels. The instrumental resolution is measured as the mean value of Gaussian FWHMs (Full Width at Half Maximum) using a number of unblended arc-lamp lines over the whole spectra range of a wavelength-calibrated spectrum. We obtain $\mathrm{FWHM}_{\text {inst }}=0.7 \AA$, corresponding to a velocity dispersion $\sigma_{\text {inst }}=19 \mathrm{~km} \mathrm{~s}^{-1}$ at 5100 $\AA$. The angular sampling is 0.252 arcsec pixel ${ }^{-1}$, with a reciprocal dispersion of $0.235 \AA$ pixel $^{-1}$. The median 
Table 1. Physical parameters of sample bulges.

\begin{tabular}{ccccc}
\hline \hline Galaxy & $r_{\mathrm{e}}$ & $\sigma_{\mathrm{e}}$ & $M_{\text {bulge }}$ & $B / D\left(r<r_{\mathrm{e}}\right)$ \\
& $(\mathrm{pc})$ & $\left(\mathrm{km} \mathrm{s}^{-1}\right)$ & $\left(\log \left(M / M_{\odot}\right)\right)$ & \\
$(1)$ & $(2)$ & $(3)$ & $(4)$ & $(5)$ \\
\hline NGC 2503 & $637 \pm 27$ & $63 \pm 6$ & $9.18 \pm 0.23$ & 3.6 \\
NGC 2684 & $225 \pm 7$ & $53 \pm 5$ & $8.34 \pm 0.22$ & 1.5 \\
NGC 2742 & $253 \pm 7$ & $53 \pm 3$ & $8.21 \pm 0.19$ & 1.7 \\
NGC 2913 & $363 \pm 11$ & $59 \pm 3$ & $8.76 \pm 0.23$ & 5.7 \\
NGC 3041 & $354 \pm 24$ & $58 \pm 3$ & $8.68 \pm 0.22$ & 2.1 \\
NGC 3055 & $168 \pm 7$ & $50 \pm 4$ & $8.20 \pm 0.21$ & 3.8 \\
NGC 3294 & $277 \pm 8$ & $76 \pm 5$ & $8.64 \pm 0.18$ & 2.8 \\
NGC 3486 & $144 \pm 4$ & $66 \pm 1$ & $8.63 \pm 0.18$ & 4.0 \\
NGC 3810 & $151 \pm 4$ & $65 \pm 1$ & $8.55 \pm 0.19$ & 1.5 \\
NGC 3982 & $117 \pm 4$ & $76 \pm 2$ & $8.39 \pm 0.19$ & 1.3 \\
NGC 4237 & $123 \pm 4$ & $55 \pm 2$ & $8.05 \pm 0.19$ & 1.0 \\
NGC 4305 & $332 \pm 22$ & $42 \pm 1$ & $8.68 \pm 0.20$ & 4.8 \\
NGC 4405 & $198 \pm 19$ & $41 \pm 3$ & $8.30 \pm 0.20$ & 0.7 \\
NGC 4598 & $431 \pm 43$ & $39 \pm 1$ & $8.80 \pm 0.20$ & 1.8 \\
NGC 4620 & $859 \pm 58$ & $38 \pm 1$ & $8.66 \pm 0.21$ & 1.6 \\
NGC 4654 & $125 \pm 13$ & $44 \pm 5$ & $8.10 \pm 0.21$ & 5.0 \\
NGC 4689 & $664 \pm 44$ & $31 \pm 4$ & $9.13 \pm 0.19$ & 1.5 \\
NGC 5480 & $267 \pm 8$ & $57 \pm 5$ & $8.61 \pm 0.21$ & 3.9 \\
NGC 5768 & $441 \pm 13$ & $64 \pm 4$ & $8.93 \pm 0.22$ & 1.8 \\
NGC 5956 & $414 \pm 27$ & $77 \pm 2$ & $9.33 \pm 0.21$ & 6.0 \\
NGC 5957 & $504 \pm 50$ & $77 \pm 4$ & $9.11 \pm 0.21$ & 3.6 \\
NGC 6106 & $465 \pm 13$ & $59 \pm 5$ & $7.85 \pm 0.21$ & 0.4 \\
NGC 7137 & $130 \pm 4$ & $24 \pm 5$ & $8.11 \pm 0.20$ & 3.2 \\
IC 0492 & $617 \pm 26$ & $86 \pm 6$ & $8.25 \pm 0.21$ & 4.9 \\
IC 1066 & $309 \pm 9$ & $58 \pm 4$ & $9.24 \pm 0.22$ & 0.5 \\
IC 2293 & $213 \pm 9$ & $20 \pm 8$ & $8.15 \pm 0.23$ & 45.3 \\
\hline
\end{tabular}

value of the seeing FWHM during the observing nights was $\sim 0^{\prime \prime} .9$.

\section{DATA ANALYSIS}

We performed standard reduction for both photometric and spectroscopic data, as thoroughly described in Costantin et al. (2017).

For all 26 galaxies, we retrieved $i$-band SDSS DR13 images, in order to properly characterize the different substructures in the galaxies (i.e., bulge, disk, and bar component). We derived the structural parameters of the sample galaxies by performing a two-dimensional photometric decomposition of their surface brightness distribution using the GAlaxy Surface Photometry 2 Dimensional Decomposition (GASP2D; Méndez-Abreu et al. 2008, 2014). In particular, this allows us to prop- erly define the half-light radius of the bulge component, which results critical in homogenizing the kinematics measurements.

Following Taylor et al. (2011), we use the empirical relation between $(g-i)$ color, $i$-band luminosity, and stellar mass

$$
\log \left(\mathrm{M}_{\star} / M_{\odot}\right)=-0.68+0.7(g-i)-0.4\left(\mathcal{M}_{i}-4.58\right),
$$

to derive the stellar mass of the bulge, where $\mathcal{M}_{i}$ corresponds to its absolute magnitude in the rest-frame $i$ band expressed in the $\mathrm{AB}$ system. The relation provides estimate to a $1 \sigma$ accuracy of $\sim 0.1$ dex and was calibrated according to Galaxy And Mass Assembly (GAMA) sample of galaxies (Driver et al. 2009).

We used the absorption features in the long-slit spectra to measured the stellar kinematics within the bulge half-light radius, adopting the penalized pixel-fitting method (Cappellari \& Emsellem 2004) and the Gas and Absorption Line Fitting algorithm (GANDALF; Sarzi et al. 2006). The observed spectra were convolved with a linear combination of stellar spectra from the ELODIE library (FWHM = 0.48 Å; Prugniel \& Soubiran 2001).

The high spectral and spatial resolution kinematics allows us to measure reliable stellar velocity dispersions within the bulges half-light radii down to $\sim 20 \mathrm{~km} \mathrm{~s}^{-1}$, pushing the limit of available instruments to a still unexplored regime. The photometric information from $i$ band SDSS images allows us to characterize bulges of typical scale of $\sim 100-800 \mathrm{pc}$ down to masses of $\sim$ $10^{8} M_{\odot}$ (see Table 1). Inside the bulge effective radius the surface brightness distribution for most of the sample galaxies is dominated by the bulge contribution $\left(B / D\left(r<r_{\mathrm{e}}\right) \gtrsim 1\right)$. Therefore, our luminosity-weighted spectroscopic measurements are probing the bulge kinematics.

Considering a possible contamination from the underling disk dynamics, to assure the reliability of the bulge velocity dispersion measurements we create mock spectra of galaxies with bulge and disk components having physical properties that resemble our sample bulges. We use the bulge half-light radius $r_{\mathrm{e}}=0.35 \mathrm{kpc}$, bulge Sérsic index $n=1.2$, disk scale length $h=3 \mathrm{kpc}$, and bulge velocity dispersion $\sigma_{\mathrm{e}}=58 \mathrm{~km} \mathrm{~s}^{-1}$. We use several velocity fields for the disk component using the rotation curve profiles in Persic et al. (1996). To test some extreme possible scenarios, we used values of disk velocities rising to more than $100 \mathrm{~km} \mathrm{~s}^{-1}$ within the bulge halflight radius. The value of the bulge velocity dispersion is reliably measured in all mock spectra, with a small bias up to $\sim 5 \mathrm{~km} \mathrm{~s}^{-1}$.

The dynamical status of the sample bulges is compared with a complementary sample of virialized stellar 
systems over seven orders of magnitude in mass, comparing their physical properties using kinematics values measured integrating the light within their half-light radius. This represents a dissimilarity compared to the majority of previous works, where the central velocity dispersion of the galaxy is used instead of the value integrated within the half-light radius due to either the lack of the photometric information or the less demanding spectroscopic data needed to retrieve the central value. The photometric decomposition of the galaxy light is indeed necessary to accurately characterize various substructures (i.e., bulges).

Firstly, we complement the sample with the seven small bulges presented in Costantin et al. (2017). Then, we measure kinematics within the half-light radius and stellar masses of massive systems like large bulges (Méndez-Abreu et al. 2017) and elliptical galaxies (Falcón-Barroso et al. 2017; Iodice et al. 2019) using integral-field data from Calar Alto Legacy Integral Field Area Survey (CALIFA; Sánchez et al. 2016) and FORNAX3D (Sarzi et al. 2018) samples, respectively. Finally, we use literature values of kinematics and stellar masses of globular clusters (GCs; Taylor et al. 2015), nuclear star clusters (NSCs; Kacharov et al. 2018), compact early-type galaxies (cETGs; Norris et al. 2014; Guérou et al. 2015), and ultra compact dwarf galaxies (UCDs; Mieske et al. 2008). When only dynamical masses were available, we used them as upper limit for the stellar mass.

\section{MASS-VELOCITY DISPERSION RELATION}

The photometric and kinematic information allows us to characterize the dynamical status of the different stellar systems, including our small bulges, by means of their positions in fundamental scaling relations. In Fig. 1 we show the stellar mass versus velocity dispersion ("mass Faber-Jackson" relation), which is a direct consequence of the Virial Theorem and represents a projection of the Fundamental Plane. This plane represents the cooling diagram in theories of galaxy formation, relating the the virial temperature and the stellar density. From a theoretical perspective, the amount of energy dissipation during the system formation and the timing at which it occurs is directly related to its position in this diagram. From the Virial Theorem, we can express the stellar luminosity as a function of the total mass as

$$
L \propto \frac{r_{\mathrm{e}} \sigma^{2}}{(M / L)_{\text {vir }}},
$$

where $r_{\mathrm{e}}$ and $\sigma$ are the half-light radius and the velocity dispersion of the galaxy, respectively. Using the average surface brightness within the half-light radius $\left(\left\langle I_{\mathrm{e}}\right\rangle\right)$, the luminosity is $L \propto\left\langle I_{\mathrm{e}}\right\rangle r_{\mathrm{e}}^{2}$. Considering that the luminosity is a proxy for the stellar mass, it is

$$
M_{\star} \propto \frac{\sigma^{4}}{\left\langle I_{\mathrm{e}}\right\rangle(M / L)_{\mathrm{vir}}^{2}},
$$

which corresponds to the "mass Faber-Jackson" relation if $\left\langle I_{\mathrm{e}}\right\rangle$ and $(M / L)_{\text {vir }}$ are constant.

We find that a tight relation (scatter of 0.15 dex) unifies large and more massive stellar systems like elliptical galaxies $\left(r_{\mathrm{e}} \gtrsim 5 \mathrm{kpc}, M_{\star}>10^{11} M_{\odot}\right)$ and large bulges $\left(r_{\mathrm{e}} \gtrsim 1 \mathrm{kpc}, M_{\star}=10^{10} M_{\odot}\right.$, mostly residing in ETGs), and small and less massive stellar systems like NSCs $\left(r_{\mathrm{e}}\right.$ $\left.\sim 10 \mathrm{pc}, M_{\star} \sim 10^{7} M_{\odot}\right)$ and GCs $\left(r_{\mathrm{e}} \sim 10 \mathrm{pc}, M_{\star} \sim\right.$ $\left.10^{6} M_{\odot}\right)$. For some systems their dynamical masses are used as upper limits for the stellar mass. Figure 1 shows that small bulges in late-type galaxies connect the previous stellar virialized systems over seven magnitudes in mass. They bridge the gap between less and more massive virialized stellar systems, sharing the same position in the plane as some compact systems like cETGs, UCDs, and massive NSCs. As a reference, we also overplot the core of the spiral galaxies M33 and M101 (Kormendy et al. 2010) and the compact elliptical galaxy M32 (Chilingarian et al. 2007), which are shown to follow the relation. M32 is the closest (low-mass) elliptical galaxy, while M33 and M101 represent two examples of nearby pure-disk galaxies (with a very small core).

Once we properly take into account the dynamical status of these systems by deriving their properties in a consistent way within the half-light radius, there is a remarkable agreement between the theoretical prediction from the Virial Theorem and our best-fit estimation:

$$
M_{\star} \propto \sigma_{\mathrm{e}}^{4.01 \pm 0.06} .
$$

Cappellari et al. (2013) calculated $M_{\text {vir }} \propto \sigma^{4.7 \pm 0.6}$ for galaxies with $\sigma \gg 140 \mathrm{~km} \mathrm{~s}^{-1}$ and $M_{\text {vir }} \propto \sigma^{2.3 \pm 0.1}$ for galaxies with $\sigma \ll 140 \mathrm{~km} \mathrm{~s}^{-1}$ in ATLAS ${ }^{3 D}$ sample. Bezanson et al. (2015) derived $M_{\star} \propto \sigma^{2.44}$ using SDSS galaxies $\left(M_{\star} \sim 10^{10-11.5} M_{\odot}\right)$, with half-light radius velocity dispersion measurements derived applying aperture correction from the $3^{\prime \prime}$ SDSS fibers. At higher redshift, analyzing a sample of DEIMOS galaxies $\left(M_{\star} \sim 10^{10-11.5} M_{\odot}\right)$ at $z \sim 0.7$, Bezanson et al. (2015) obtained $M_{\star} \propto \sigma^{2.94}$. All these results agree with Balcells et al. (2007) $\left(L \propto \sigma^{2.9 \pm 0.5}\right)$ and Costantin et al. (2017) $\left(L \propto \sigma^{2.64 \pm 0.01}\right)$. We suggest these differences from the theoretical prediction are due to either the small mass range explored or the not homogeneous/reliable comparison sample. By exploring a wider mass regime as we have done for the first time in this work, which also requires accurate kinematic measurements, we replicate the Virial Theorem prediction de- 


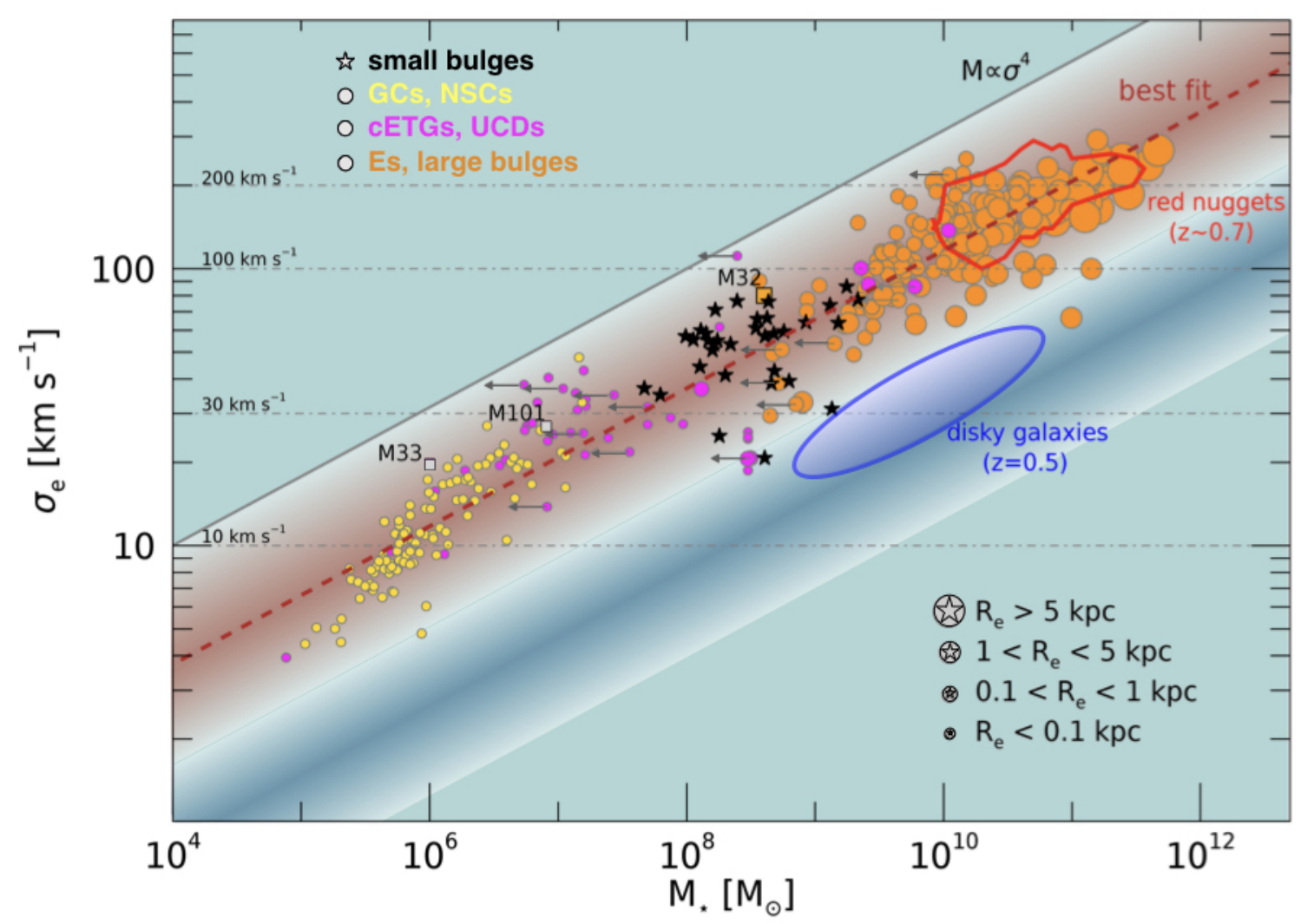

Figure 1. Virial stellar mass versus velocity dispersion ("mass Faber-Jackson" relation) relation for small (globular clusters and nuclear stellar clusters; yellow circles), compact (compact early type galaxies and ultra compact dwarfs; purple circles), and large (ellipticals and large bulges; orange circles) systems. Observations of small bulges are shown as black stars. The best-fit relation (dark red dashed line) and the virial $M \propto \sigma^{4}$ relation (gray solid line) are shown. The symbol size represents the effective radius of each system. The red shadow represents virialized stellar systems, where the red contour marks the region of massive compact systems population (i.e., red nuggets) measured at redshift $z \sim 0.7$. The blue shadow stands for the off-set sequence expected for disk-like stellar systems, where an example at redshift $z=0.5$ from Illustris TNG50 simulations is shown as blue contour. As a reference, the core of the spiral galaxies M33 and M101 and the compact elliptical galaxy M32 are superimposed to the main relation (squares). Gray arrows stand for systems where dynamical masses are computed, which represent an upper limit for the stellar mass.

scribing the physical resemblance between all these virialized systems in the "mass Faber-Jackson" relation.

Finally, we compare the kinematics of the virialized systems with the one of disk galaxies at $z=0.5$, as predicted from the state-of-the-art hydrodynamical simulations (Illustris TNG50; Pillepich et al. 2019). Disk galaxies are offset from the virial relation, ruling out the possibility that the dynamical status of our small bulges resembles the one of disk-like systems.

\section{DISCUSSION AND CONCLUSIONS}

The relation between fundamental galactic dynamical and structural properties, which manifests in global scaling laws, carries important clues about the process of galaxy formation in a cosmological context. Interpreting the phenomenology of these relations can indeed have substantial consequences on our understanding of the interplay between baryons and dark matter, including its varying nature in the $\Lambda$ CDM paradigm.

We find that stellar systems over seven orders of magnitude in mass follow the same fundamental "mass Faber-Jackson" relation, which directly follows from the Virial Theorem prediction. This result is achieved by homogeneously comparing systems whose physical properties are measured within their half-light radius using reliable spectroscopic information. While the observed trend is not surprising for elliptical galaxies and massive bulges, we reveal that small bulges are not offset from this relation and follow the $M_{\star} \propto \sigma^{4}$ prediction from the Virial Theorem. This leads to our new interpretation that small bulges in the center of today's extremely late-type galaxies maintain memory of an ancient formation, as virialized systems do. Moreover, small bulges share the same position in the "mass Faber-Jackson" 
plane as cETGs, UCDs, and massive NSCs. This further support our proposed scenario, because (at least) the most massive cETGs, UCDs, and GCs (e.g., $\omega$ Cen in the Milky Way) have been proposed to be linked to the remnants of tidally disrupted nucleated galaxies (Drinkwater et al. 2003), which represent the first generation of cluster satellites and are the result of an early and rapid formation (Sánchez-Janssen et al. 2019). This early formation of the central region of late-type galaxies alleviate the tension between SMBH and bulge coevolution at high redshift. Thus, small bulges should not represent an exception of the fundamental scaling relations that regulate $\mathrm{SMBH}$ and bulge interplay, even though the progressively lower binding energies of lowmass galaxies intuitively allow for a different regulation of SMBH and bulge synergic growth.

Recently, using N-body simulations Guo et al. (2019) have shown that the destruction of short bars due to the presence of SMBH of $\sim 0.1 \%$ the stellar mass in galaxies of $M_{\star} \sim 4 \times 10^{10} M_{\odot}$, could end up mimicking the photometric and kinematic properties of virialized bulges. However, the fraction of galaxies experiencing the different phases required by this scenario (early inner disk formation, short bar formation, and destruction) is difficult to estimate with the available observations and simulations.

On the other hand, the scenario proposed by Hopkins et al. (2009) postulates that the massive highredshift quiescent compact galaxies (red nuggets; Damjanov et al. 2009) end up in the center of present-day massive elliptical galaxies. This traditional evolutionary picture leads to extend the paradigm to disk galaxy bulges (Graham \& Scott 2013; de la Rosa et al. 2016). In the $\Lambda$ CDM context, Zolotov et al. (2015) used cosmological simulations to show that after compaction extended star-forming disks can develop around red nuggets. It is worth noting that the velocity dispersion of the central component in galaxies is relatively unaffected by the loss of an outer stellar or dark matter halo (Chilingarian et al. 2009), and seems also not to be perturbed by the growth of the extended disk component (Debattista et al. 2013). The results presented in this work support the idea that also late-type galaxies might host compact stellar systems in their central region, providing a general and broader picture of galaxy evolution at all masses in an inside-out scenario. Therefore, to further investigate a possible connection between galaxies in the Local universe and their progenitors, we overplot in Fig. 1 a sample representing the red nugget population observed by Bezanson et al. (2015) at $z \sim 0.7$, showing that they occupy the massive end of the "mass Faber-Jackson" relation. In this view, we speculate that not only the center of ETGs should hide the remnants of the compact objects population observed at high redshift, but also the core of late-type galaxies could represent the low-mass tail of high-redshift formed red nuggets.

\section{ACKNOWLEDGMENTS}

We would like to thank the anonymous referee for improving the content of the manuscript. LC wishes to thank CCG for inspiration and AC for the useful discussion while this manuscript was written. LC acknowledges financial support from Comunidad de Madrid under Atracción de Talento grant 2018-T2/TIC-11612 and the Spanish Ministerio de Ciencia, Innovacin y Universidades through grant PGC2018-093499-B-I00. JMA and AdLC acknowledge support from the Spanish Ministerio de Economia y Competitividad (MINECO) grants AYA2017-83204-P and AYA201677237-C3-1-P, respectively. EMC is supported by MIUR grant PRIN 2017 20173ML3WW_001. IP is supported by Leibniz-Institut fur Astrophysik Potsdam. VC and IP acknowledge support from the Fondazione Ing. Aldo Gini. EMC, VC, and MR are funded by Padua University grants DOR1715817/17, DOR1885254/18, and DOR1935272/19.

\section{REFERENCES}

Albareti, F. D., Allende Prieto, C., Almeida, A., et al. 2017, ApJS, 233, 25, doi: 10.3847/1538-4365/aa8992

Balcells, M., Graham, A. W., \& Peletier, R. F. 2007, ApJ, 665, 1104, doi: 10.1086/519753

Bezanson, R., Franx, M., \& van Dokkum, P. G. 2015, The Astrophysical Journal, 799, 148, doi: 10.1088/0004-637X/799/2/148

Bullock, J. S., \& Boylan-Kolchin, M. 2017, ARA\&A, 55, 343, doi: 10.1146/annurev-astro-091916-055313

Cappellari, M., \& Emsellem, E. 2004, PASP, 116, 138, doi: $10.1086 / 381875$
Cappellari, M., McDermid, R. M., Alatalo, K., et al. 2013, MNRAS, 432, 1862, doi: 10.1093/mnras/stt644

Chilingarian, I., Cayatte, V., Chemin, L., et al. 2007, A\&A, 466, L21, doi: 10.1051/0004-6361:20077291

Chilingarian, I., Cayatte, V., Revaz, Y., et al. 2009, Science, 326, 1379, doi: 10.1126/science.1175930

Costantin, L., Méndez-Abreu, J., Corsini, E. M., et al. 2017, Astronomy and Astrophysics, 601, A84, doi: 10.1051/0004-6361/201630302

Damjanov, I., McCarthy, P. J., Abraham, R. G., et al. 2009, ApJ, 695, 101, doi: 10.1088/0004-637X/695/1/101 
de la Rosa, I. G., La Barbera, F., Ferreras, I., et al. 2016, MNRAS, 457, 1916, doi: 10.1093/mnras/stw130

Debattista, V. P., Kazantzidis, S., \& van den Bosch, F. C. 2013, ApJ, 765, 23, doi: 10.1088/0004-637X/765/1/23

Dekel, A., Sari, R., \& Ceverino, D. 2009, ApJ, 703, 785, doi: 10.1088/0004-637X/703/1/785

Djorgovski, S., \& Davis, M. 1987, The Astrophysical Journal, 313, 59, doi: 10.1086/164948

Drinkwater, M. J., Gregg, M. D., Hilker, M., et al. 2003, Nature, 423, 519, doi: 10.1038/nature01666

Driver, S. P., Norberg, P., Baldry, I. K., et al. 2009, Astronomy and Geophysics, 50, 5.12, doi: 10.1111/j.1468-4004.2009.50512.x

Falcón-Barroso, J., Lyubenova, M., van de Ven, G., et al. 2017, Astronomy and Astrophysics, 597, A48, doi: 10.1051/0004-6361/201628625

Ferrarese, L., \& Merritt, D. 2000, ApJL, 539, L9, doi: 10.1086/312838

Fisher, D. B., \& Drory, N. 2016, in Astrophysics and Space Science Library, Vol. 418, Galactic Bulges, ed. E. Laurikainen, R. Peletier, \& D. Gadotti, 41, doi: 10.1007/978-3-319-19378-6_3

Graham, A. W., \& Scott, N. 2013, ApJ, 764, 151, doi: 10.1088/0004-637X/764/2/151

Guérou, A., Emsellem, E., McDermid, R. M., et al. 2015, The Astrophysical Journal, 804, 70, doi: 10.1088/0004-637X/804/1/70

Guo, M., Du, M., Ho, L. C., Debattista, V. P., \& Zhao, D. 2020, ApJ, 888, 65, doi: 10.3847/1538-4357/ab584a

Hopkins, P. F., Bundy, K., Murray, N., et al. 2009, MNRAS, 398, 898, doi: 10.1111/j.1365-2966.2009.15062.x

Iodice, E., Sarzi, M., Bittner, A., et al. 2019, Astronomy and Astrophysics, 627, A136, doi: 10.1051/0004-6361/201935721

Kacharov, N., Neumayer, N., Seth, A. C., et al. 2018, Monthly Notices of the Royal Astronomical Society, 480, 1973, doi: 10.1093/mnras/sty1985

Kormendy, J., \& Bender, R. 2011, Nature, 469, 377, doi: 10.1038/nature09695

Kormendy, J., Bender, R., \& Cornell, M. E. 2011, Nature, 469, 374, doi: 10.1038/nature09694

Kormendy, J., Drory, N., Bender, R., \& Cornell, M. E. 2010, The Astrophysical Journal, 723, 54, doi: 10.1088/0004-637X/723/1/54

Kormendy, J., \& Fisher, D. B. 2008, in Astronomical Society of the Pacific Conference Series, Vol. 396, Formation and Evolution of Galaxy Disks, ed. J. G. Funes \& E. M. Corsini, 297
Kormendy, J., \& Kennicutt, Jr., R. C. 2004, ARA\&A, 42, 603, doi: 10.1146/annurev.astro.42.053102.134024

Méndez-Abreu, J., Aguerri, J. A. L., Corsini, E. M., \& Simonneau, E. 2008, A\&A, 478, 353, doi: 10.1051/0004-6361:20078089

Méndez-Abreu, J., Debattista, V. P., Corsini, E. M., \& Aguerri, J. A. L. 2014, A\&A, 572, A25, doi: 10.1051/0004-6361/201423955

Méndez-Abreu, J., Ruiz-Lara, T., Sánchez-Menguiano, L., et al. 2017, Astronomy and Astrophysics, 598, A32, doi: 10.1051/0004-6361/201629525

Mieske, S., Hilker, M., Jordán, A., et al. 2008, Astronomy and Astrophysics, 487, 921, doi: 10.1051/0004-6361:200810077

Norris, M. A., Kannappan, S. J., Forbes, D. A., et al. 2014, Monthly Notices of the Royal Astronomical Society, 443, 1151, doi: 10.1093/mnras/stu1186

Oser, L., Ostriker, J. P., Naab, T., Johansson, P. H., \& Burkert, A. 2010, ApJ, 725, 2312, doi: 10.1088/0004-637X/725/2/2312

Persic, M., Salucci, P., \& Stel, F. 1996, MNRAS, 281, 27, doi: 10.1093/mnras/281.1.27

Pillepich, A., Nelson, D., Springel, V., et al. 2019, MNRAS, 490, 3196, doi: 10.1093/mnras/stz2338

Prugniel, P., \& Soubiran, C. 2001, A\&A, 369, 1048, doi: 10.1051/0004-6361:20010163

Sánchez, S. F., García-Benito, R., Zibetti, S., et al. 2016, A\&A, 594, A36

Sánchez-Janssen, R., Côté, P., Ferrarese, L., et al. 2019, ApJ, 878, 18, doi: 10.3847/1538-4357/aaf4fd

Sarzi, M., Falcón-Barroso, J., Davies, R. L., et al. 2006, MNRAS, 366, 1151, doi: 10.1111/j.1365-2966.2005.09839.x

Sarzi, M., Iodice, E., Coccato, L., et al. 2018, A\&A, 616, A121, doi: 10.1051/0004-6361/201833137

Silk, J., \& Rees, M. J. 1998, A\&A, 331, L1

Taylor, E. N., Hopkins, A. M., Baldry, I. K., et al. 2011, MNRAS, 418, 1587, doi: 10.1111/j.1365-2966.2011.19536.x

Taylor, M. A., Puzia, T. H., Gomez, M., \& Woodley, K. A. 2015, The Astrophysical Journal, 805, 65, doi: 10.1088/0004-637X/805/1/65

White, S. D. M., \& Rees, M. J. 1978, Monthly Notices of the Royal Astronomical Society, 183, 341, doi: 10.1093/mnras/183.3.341

Zolotov, A., Dekel, A., Mandelker, N., et al. 2015, MNRAS, 450, 2327, doi: 10.1093/mnras/stv740 